\title{
Modelagem de Sistema de Comunicação com Modulação Digital baseada em Quatérnios
}

\author{
Juan Carlos Oliveira de Medeiros, Diego Aguiar Sousa e João César Moura Mota
}

\begin{abstract}
Resumo-Este artigo apresenta o uso da álgebra dos Quatérnios, com suas vantagens computacionais em função da redução da quantidade de operações algébricas envolvendo produtos reais usuais indo de encontro com o modelo matricial. Nós propomos um novo esquema de modulação, denominado QuatMod, em sistemas de comunicação digital com uma nova formulação analítica para análise de Probabilidade de erro em um canal com AWGN quaterniônico equivalente. A modulação QuatMod é realizada com constelações quadrangulares, geometricamente representadas via isomorfismo por classes de hipercubos em $\mathbb{R}^{4}$. Nós usamos a representação Cayley-Dickson, interpretada por uma dada estrutura quaterniônica como uma portadora equivalente para dois números complexos. Ela é aplicada na transmissão entre duas antenas de polarização dupla, refletindo em uma comparação com a abordagem clássica já existente. Por fim, o nosso trabalho também traz um estudo acerca da modelagem de um canal quaterniônico com o modelo clássico.
\end{abstract}

Palavras-Chave-Quatérnios, Modulação, Canal, Polarização.

Abstract-This paper presents the Quarternions algebraic and their computational advantages in function of the decreasing of the algebraic operations involving the usuals real products going against the matrix model. We propose a new modulation scheme, called QuatMod, for the digital communications systems and a new analytical formulation for the error probability for an AWGN quaternionic equivalent channel. The QuatMod is performed for quadrangular constellations represented geometrically by isomorphism by hypercubes classes in $\mathbb{R}^{4}$. We used the Cayley-Dickson representation, interpreted by a given quaternionic structure as an equivalent carrier for two complex numbers. This representation is applied in the transmission between two dual polarized antennas, reflecting in a comparison with the existing classic approach. Finally, we also study de quarternionic channel modeling by comparing with the classic model.

Keywords-Quaternions, Modulation, Channel, Polarization.

\section{INTRODUÇÃo}

Durante os últimos anos, tem-se notado um grande avanço tecnológico na área de sistemas de comunicações sem fio, desde os antigos sistemas analógicos de primeira geração, até os sistemas digitais mais modernos presentes atualmente. Estes avanços vêm ocorrendo em diversos campos de estudo dos sistemas de telecomunicações, tais como no processamento digital de sinais, codificação de canal e sinal, modulação e multiplexação de informação, dentre vários outros.

Tais avanços tecnológicos, aliado à praticidade de uso de equipamentos de comunicação sem fio em detrimento

Dpto. Eng. Teleinformática. Univ. Federal do Ceará, Fortaleza-CE, Brasil E-mails: juca@ufc.br, diego@gtel.ufc.br, mota@gtel.ufc.br. Este trabalho foi parcialmente financiado pelo CNPq e pela FUNCAP. a comunicação cabeada, têm motivado, nos últimos anos, o crescimento do número de equipamentos, usuários e tecnologias que utilizam esse meio de comunicação. Aliado a este crescimento, observa-se também uma maior demanda por taxas de transmissão maiores e melhor qualidade de transmissão. No entanto, para que tais demandas sejam alcançadas é necessário pesquisar por formas de uso mais eficientes do espectro eletromagnético, visto que o mesmo possui uma banda utilizável bastante estreita frente as necessidades atuais. Neste contexto, várias técnicas de multiplexação de sinais vem sendo estudadas, dentre elas encontra-se a multiplexação espacial de sinais utilizando a diversidade de polarização [1], [2].

Esta técnica consiste em transmitir os sinais através de antenas operando em campos elétricos com diferentes polarizações, de forma não correlacionada, se mostrando consideravelmente mais eficiente que as técnicas de diversidade espacial convencionais em ambientes densos, com uma grande quantidade de multipercursos. Ainda neste contexto, o uso de diversidade por polarização implica em uma redução no tamanho das antenas, além de as mesmas poderem ser instaladas bastante próximas umas as outras, o que na diversidade espacial tradicional é raramente viável [3].

Outro desafio dos sistemas de comunicação sem fio, principalmente para a telefonia móvel, é o aumento da vida útil dos equipamentos e o uso eficiente da bateria. Uma das maneiras de se alcançar este objetivo é diminuir o número de operações computacionais envolvidas no processamento dos sinais nos equipamentos. Neste contexto, alguns trabalhos vêm introduzindo o estudo os quatérnios em sistemas de comunicação. Os quatérnios são um conjunto numérico matemático que representam uma extensão dos números complexos. Eles são bastante utilizados na área da computação gráfica por conta de suas propriedades algébricas favoráveis e suas vantagens computacionais em relação às operações matriciais [9].

Em teoria de sinais, os quatérnios foram abordados no início dos anos 1990, por [4], que introduziu a transformada quaterniônica no estudo de sinais hipercomplexos. Já na década passada, em [5] foi realizado uma implementação eficiente da transformada de Fourier, da convolução e da correlação quaterniônica, através do uso da transformada rápida de Fourier (FFT, Fast Fourier Transform) bidimensional. Em [6], os autores apresentaram um modelo de processamento de sinal de três dimensões utilizando quatérnios. Já em [7], foi mostrado um modelo de transmissão quaterniônico equivalente a um modelo de transmissão MIMO com 2 antenas dual polarizadas no transmissor e no receptor. 
Por fim, em [8], os autores desenvolveram técnicas para a construção de códigos ortogonais espaço-temporais quaterniônicos de bloco. Atualmente, há alguns trabalhos referenciando o uso de quatérnios em processamento de sinal, como por exemplo: decomposição de valores singulares [10], método MUSIC - MUltiple SIgnal Classification para estimativa de espectro [11], filtragem adaptativa [12], propriedades estátisticas quaterniônicas [13], tratamento de auto regressão quaterniônica[14], entre outros.

Neste trabalho, objetiva-se generalizar o modelo apresentado em [7], bem como desenvolver uma modulação de símbolos quaterniônica adequada para o modelo de canal quaterniônico apresentado. Além disso, uma formulação analítica para a probabilidade de erro neste sistema será desenvolvida para um canal contaminado com ruído Gaussiano branco (AWGN, Additive White Gaussian Noise).

O restante deste artigo está dividido da seguinte forma: $\mathrm{Na}$ seção II são apresentados alguns conceitos básicos acerca do conjunto dos quatérnios que serão utilizados no decorrer do trabalho. Na seção III é apresentado o modelo sistêmico utilizado neste trabalho. Na seção IV é desenvolvida uma nova técnica de modulação de informação quaterniônica. Além disso, nesta seção também será desenvolvida uma formulação analítica para um canal AWGN. Na seção V serão apresentados os resultados de simulação comprovando os resultados teóricos anteriormente apresentados. E, por fim, na seção VI serão apresentadas as conclusões deste trabalho, bem como as perspectivas de trabalhos futuros.

\section{CONCEITOS BÁSICOS SOBRE QuATÉRnios}

$\mathrm{O}$ conjunto dos quatérnios é denotado por $\mathbb{H}$, em homenagem Hamilton. Um quatérnio $q$ consiste de uma parte escalar $s \in \mathbb{R}$ e de um vetor $\mathbf{v}=(x, y, z) \in \mathbb{R}^{3}$. Sejam $\mathbf{i}^{2}=\mathbf{j}^{2}=\mathbf{k}^{2}=-1, \mathbf{i j}=\mathbf{k}, \mathbf{j i}=-\mathbf{k}, \mathbf{j k}=\mathbf{i}, \mathbf{k j}=-\mathbf{i}$, $\mathbf{k i}=\mathbf{j}$ e $\mathbf{i} \mathbf{k}=-\mathbf{j}$, então as representações de um elemento $q \in \mathbb{H}$ podem ser escritas como

$$
\begin{array}{rlrl}
q & \equiv[s, \mathbf{v}] & & \text { para } s \in \mathbb{R}, \mathbf{v} \in \mathbb{R}^{3} \\
& \equiv[s,(x, y, z)] & & \text { para } s, x, y, z \in \mathbb{R} \\
& \equiv s+x \mathbf{i}+y \mathbf{j}+z \mathbf{k} & \text { para } s, x, y, z \in \mathbb{R}
\end{array}
$$

Sejam $q_{1}, q_{2} \in \mathbb{H}$ com $q_{1}=\left[s_{1},\left(x_{1}, y_{1}, z_{1}\right)\right]$ e $q_{2}=$ $\left[s_{2},\left(x_{2}, y_{2}, z_{2}\right)\right]$. Então o operador de adição, $(+)$, é definido como

$$
q_{1}+q_{2}=\left[s_{1}+s_{2},\left(\left(x_{1}+x_{2}\right),\left(y_{1}+y_{2}\right),\left(z_{1}+z_{2}\right)\right)\right] .
$$

Desta forma o operador de adição 2 está bem definido e, é comutativo, ou seja, $q_{1}+q_{2}=q_{2}+q_{1}$. Além disso, pode-se definir, como em [15], o operador de multiplicação entre dois quatérnios, $(\cdot)$, como sendo da seguinte forma

$$
q_{1} \cdot q_{2}=\left[s_{1} s_{2}-\left\langle\mathbf{v}_{1}, \mathbf{v}_{2}\right\rangle, \mathbf{v}_{1} \times \mathbf{v}_{2}+s_{1} \mathbf{v}_{2}+s_{2} \mathbf{v}_{1}\right],
$$

em que $\left\langle\mathbf{v}_{1}, \mathbf{v}_{2}\right\rangle$ e $\mathbf{v}_{1} \times \mathbf{v}_{2}$ representam o produto interno e o produto vetorial usual de $\mathbb{R}^{3}$, respectivamente.

Observação 1: O conjunto dos Quatérnios não é um conjunto comutativo com relação à multiplicação, pois $\mathbf{i} \cdot \mathbf{j}=$ $\mathbf{k}$, porém $\mathbf{j} \cdot \mathbf{i}=-\mathbf{k}$.

Com respeito a operação definida em (3), a mesma apresenta de forma usual 16 operações de produtos, desempenho superior ao de matrizes $3 \times 3$ por exemplo, em que a quantidade de produtos é 27. Contudo em [9], através de $q_{1}=\left[s_{1},\left(x_{1}, y_{1}, z_{1}\right)\right]$ e $q_{2}=\left[s_{2},\left(x_{2}, y_{2}, z_{2}\right)\right]$, fazendo

$$
\begin{aligned}
& a_{1}=\left(s_{1}-x_{1}\right)\left(x_{2}-y_{2}\right) \quad a_{5}=\left(y_{1}-s_{1}\right)\left(s_{2}-x_{2}\right) \\
& a_{2}=\left(z_{1}+s_{1}\right)\left(z_{2}+s_{2}\right) \quad a_{6}=\left(y_{1}+s_{1}\right)\left(s_{2}+x_{2}\right) \\
& a_{3}=\left(z_{1}-s_{1}\right)\left(x_{2}+y_{2}\right) \quad a_{7}=\left(z_{1}+x_{1}\right)\left(z_{2}-y_{2}\right) \\
& a_{4}=\left(y_{1}+x_{1}\right)\left(z_{2}-s_{2}\right) a_{8}=\left(z_{1}-x_{1}\right)\left(z_{2}+y_{2}\right) \text {, } \\
& \text { representado por um quatérnio equivalente (4): } \\
& q_{1} \cdot q_{2}=\left(a_{1}+d-c\right)+\left(a_{2}+d-c\right) \mathbf{i}+ \\
& \left(a_{3}+d-a_{8}\right) \mathbf{j}+\left(a_{4}+d-a_{7}\right) \mathbf{k}
\end{aligned}
$$$$
c=a_{6}+a_{7}+a_{8} \text { e } d=\left(a_{5}+c\right) / 2 \text {, o produto (3) será }
$$

de modo que a redução de produtos será da ordem de 8 operações, aumentado o desempenho computacional.

Seja $q=[s, \mathbf{v}] \in \mathbb{H}$. Define-se o conjugado de $q$, denotado por $q^{*}$, como sendo

$$
q^{*} \equiv[s, \mathbf{v}]^{*} \equiv[s,-\mathbf{v}] .
$$

Com a noção de conjugado em $\mathbb{H}$, define-se a norma de um elemento $q \in \mathbb{H}$, denotada por $\|q\|_{\mathbb{H}}$, através da aplicação

$$
\begin{aligned}
\|\cdot\|_{\mathbb{H}}: \mathbb{H} & \longrightarrow \mathbb{R} \\
& q \longmapsto\|q\|_{\mathbb{H}}=\sqrt{q \cdot q^{*}}
\end{aligned}
$$

Além disso, $\|\cdot\|_{\mathbb{H}}$ representa uma norma no sentido matemático que, sem perda de generalidade, será denotado por $\|\cdot\|$. O produto interno entre dois quatérnios, $q_{1}=\left[s_{1},\left(x_{1}, y_{1}, z_{1}\right)\right]$ e $q_{2}=\left[s_{2},\left(x_{2}, y_{2}, z_{2}\right)\right]$ é definido pela seguinte aplicação

$$
\left(q_{1}, q_{2}\right) \longmapsto\left\langle q_{1}, q_{2}\right\rangle_{\mathbb{H}}=s_{1} s_{2}+x_{1} x_{2}+y_{1} y_{2}+z_{1} z_{2} .
$$

Note que, através da definição de produto interno para quatérnios, pode-se verificar que $\left\langle q_{1}, q_{1}\right\rangle_{\mathbb{H}}=s_{1}^{2}+x_{1}^{2}+y_{1}^{2}+z_{1}^{2}$. Daí, a norma de um quatérnio $q_{1}$ pode ser reescrita da seguinte forma

$$
\left\|q_{1}\right\|_{\mathbb{H}}=\sqrt{\left\langle q_{1}, q_{1}\right\rangle_{\mathbb{H}}}
$$

Observação 2: Dado um quatérnio $q=[s,(x, y, z)]$, pode-se identificar $q$ como sendo um vetor $p \in \mathbb{R}^{4}$, ou seja, $p=(s, x, y, z)$. Assim, a norma definida acima coincide com a norma Euclidiana usual de $\mathbb{R}^{4}$. Além disso, é possível representar um determinado quatérnio $q=s+x \mathbf{i}+y \mathbf{j}+z \mathbf{k}$ em duas partes, escalar e vetorial, da seguinte forma [4]:

\begin{tabular}{|l|l|}
\hline escalar & $S(q)=s$ \\
\hline vetorial & $V(q)=x \mathbf{i}+y \mathbf{j}+z \mathbf{k}$ \\
\hline
\end{tabular}

Seja $q \in \mathbb{H}$, dado que $\|q\|=1$, então $q$ é dito um quatérnio unitário. O conjunto de todos os quatérnios unitários é denotado por $\mathbb{H}_{1}$. Geometricamente, $\mathbb{H}_{1}$ é isomorfo a uma esfera unitária no espaço quadrimensional $\mathbb{R}^{4}$.

Seja $q$ um quatérnio unitário e com $S(q)=s=0$, conhecido como quatérnio puro e unitário, cuja relação de pertinência e notação de conjunto é dada por

$$
q \in P \mathbb{H}_{1}=\left\{q \in \mathbb{H}_{1}: S(q)=0\right\}
$$

com $P \mathbb{H}_{1}$ sendo chamado de conjunto dos quatérnios puros e unitários. Então

$$
\|q\|_{\mathbb{H}}=\|x \mathbf{i}+y \mathbf{j}+z \mathbf{k}\|_{\mathbb{H}}=1
$$


Por outro lado, como $q^{*}=-q=q^{-1}$, segue que

$$
\begin{aligned}
q \cdot q & =\left(-q^{*}\right) \cdot q \\
& =-\left(q^{*} \cdot q\right) \\
& =-\|q\|_{\mathbb{H}}^{2} \\
q^{2} & =-1
\end{aligned}
$$

Seja uma função $\varphi_{q}$ entre os anéis $\mathbb{C}$ e $\mathbb{H}$, da seguinte forma

$$
\begin{aligned}
\varphi_{q}: \mathbb{C} & \longrightarrow \mathbb{H} \\
a+b \mathbf{i} & \longmapsto \varphi_{q}(a+b \mathbf{i})=a+b q
\end{aligned}
$$

A função $\varphi_{q}$ é um homomorfismo entre anéis e é injetiva. Neste sentido, o corpo $\mathbb{C}$ pode ser imerso em $\mathbb{H}$ como subálgebra através de $\varphi_{q}$. E mais, pode-se definir uma classe de números quaterniônicos que comutam com respeito ao produto em $\mathbb{H}$. Com efeito,

$$
\begin{aligned}
& \varphi_{q}\left(\left(a_{1}+b_{1} \mathbf{i}\right) \cdot\left(a_{2}+b_{2} \mathbf{i}\right)\right)=\varphi_{q}\left(a_{1}+b_{1} \mathbf{i}\right) \cdot \varphi_{q}\left(a_{2}+b_{2} \mathbf{i}\right) \\
& \varphi_{q}\left(\left(a_{2}+b_{2} \mathbf{i}\right) \cdot\left(a_{1}+b_{1} \mathbf{i}\right)\right)=\varphi_{q}\left(a_{2}+b_{2} \mathbf{i}\right) \cdot \varphi_{q}\left(a_{1}+b_{1} \mathbf{i}\right)
\end{aligned}
$$

uma vez que o produto em $\mathbb{C}$ é comutativo e $\varphi_{q}$ é um homomorfismo. De modo semelhante e particular, pode-se escolher $q=\mathbf{j} \in \mathbb{H}_{1}$, puro e definir uma função $\psi_{\mathbf{j}}$ entre $\mathbb{C} \times \mathbb{C}$ e $\mathbb{H}$, definida por

$$
\begin{array}{llll}
\psi_{\mathbf{j}}: & \mathbb{C} \times \mathbb{C} & \longrightarrow \mathbb{H} \\
& \left(z_{1}, z_{2}\right) & \longmapsto & \psi_{\mathbf{j}}\left(z_{1}, z_{2}\right)=z_{1}+z_{2} \mathbf{j}
\end{array}
$$

na qual $z_{1}, z_{2} \in \mathbb{C}$, com $z_{1}=a_{1}+b_{1}$ i e $z_{2}=a_{2}+b_{2}$ i. O núcleo de $\psi_{\mathbf{j}}$ contém apenas o $(0,0) \in \mathbb{C} \times \mathbb{C}$, implicando na injetividade de $\psi_{\mathbf{j}}$ e claramente $\psi_{\mathbf{j}}(\mathbb{C} \times \mathbb{C})=\mathbb{H}$, ou seja, ela é sobrejetiva. Portanto, tem-se um isomorfismo entre $\mathbb{C} \times \mathbb{C}=\mathbb{C}^{2}$ e $\mathbb{H}$, denotado por $\mathbb{C}^{2} \simeq \mathbb{H}$. Um elemento da imagem de $\psi_{\mathbf{j}}$ é conhecido como um quatérnio na representação de Cayley-Dickson, de modo que agora pode-se pensar em um número quaterniônico como um par de números complexos. Esta representação será a base para a proposta de modulação quaterniônica que irá ser definida mais adiante.

\section{Modelo De Sistema QuATERniônico}

O modelo a ser considerado neste trabalho será o mesmo que foi apresentado em [7]. Neste modelo é considerada uma antena dual-polarizada, ou seja, uma antena que pode separar as polarizações horizontais e verticais do sinal, no transmissor e no receptor. Os sinais $s_{1}$ e $s_{2}$ são transmitidos e recebidos após a passagem pelo canal no receptor como $r_{1}$ e $r_{2}$, onde $s_{1}, s_{2}, r_{1}$ e $r_{2} \in \mathbb{C}$. Na Fig. 1 é apresentado o modelo de sistema considerado.

Assim, o sistema pode ser representado matricialmente como

$$
\left[\begin{array}{l}
r_{1} \\
r_{2}
\end{array}\right]=\left[\begin{array}{ll}
h_{h h} & h_{v h} \\
h_{h v} & h_{v v}
\end{array}\right] \cdot\left[\begin{array}{l}
s_{1} \\
s_{2}
\end{array}\right]+\left[\begin{array}{l}
n_{1} \\
n_{2}
\end{array}\right]
$$

em que $n_{1}$ e $n_{2}$ representam ruídos AWGN inseridos no receptor e cada um dos canais, $h_{h h}, h_{v h}, h_{h v}$ e $h_{v v}$, é considerado plano em frequência e possui um coeficiente complexo.

Conforme mostrado em [7], o sistema acima mostrado pode ser representado na forma quaterniônica [18] como sendo

$$
r_{q}=s_{q} \cdot q+n_{q}
$$

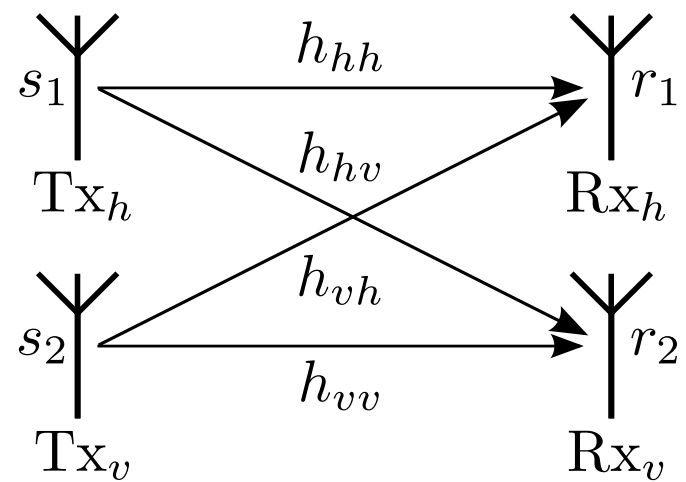

Fig. 1. Modelo de Sistema Tradicional

em que $s_{q}$ e $r_{q}$ representam os sinais transmitidos e recebidos, respectivamente, na forma quaterniônica, que são obtidos a partir da representação de Cayley-Dickson de suas representações complexas, ou seja, $s_{q}=\varphi_{\mathbf{j}}\left(s_{1}, s_{2}\right)$ e $r_{q}=$ $\varphi_{\mathbf{j}}\left(r_{1}, r_{2}\right), n_{q}$ representa o ruído AWGN em sua forma quaterniônica e $q$ denota a representação do canal no domínio dos quatérnios, dado que:

$$
\left\{\begin{array}{l}
q_{1}=\operatorname{Re}\left\{h_{h h}\right\}=\operatorname{Re}\left\{h_{v v}\right\} \\
q_{2}=\operatorname{Im}\left\{h_{h h}\right\}=-\operatorname{Im}\left\{h_{v v}\right\} \\
q_{3}=\operatorname{Re}\left\{h_{h v}\right\}=-\operatorname{Re}\left\{h_{v h}\right\} \\
q_{4}=\operatorname{Im}\left\{h_{h v}\right\}=\operatorname{Im}\left\{h_{v h}\right\}
\end{array}\right.
$$

Como se pode perceber em (12), para que o sistema representado na Fig. 1 possa ser representado em um modelo quaterniônico equivalente é necessário que $h_{h h}=h_{v v}^{*}$ e $h_{h v}=$ $-h_{v h}^{*}$. No entanto, tal suposição é perfeitamente válida para associações de antenas dual-polarizadas, devido à natureza dos canais polarizados [19], [20].

Assim, o sistema mostrado na Fig. 1 pode ser abstraído para um sistema com uma única antena no transmissor e uma no receptor, como mostrado na Fig. 2.

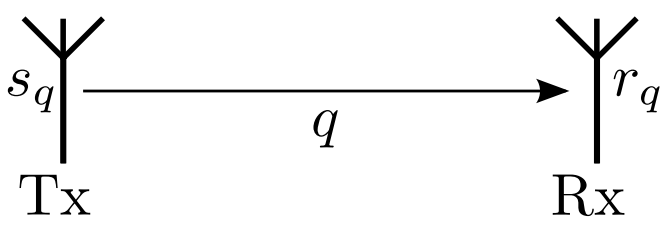

Fig. 2. Modelo de Sistema de transmissão quaterniônico

\section{MOdUlaÇÃO QUATERNIÔNICA}

A probabilidade de erro de símbolo de um sistema depende do tipo de alfabeto adotado e do nível de ruído do sinal. Por outro lado, a escolha da constelação na modulação depende do espaço e da geometria. No modelo considerado, será proposto um esquema de modulação em $\mathbb{H}$ usando o isomorfismo com $\mathbb{R}^{4}$, de modo que o conjunto de símbolos quaterniônicos sejam configurados em uma classe de hipercubos quadrimensionais. Essa nova técnica de modulação será chamada de QuatMod.

\section{A. O Modelo QuatMod}

Seja $k \in \mathbb{N}$ o número de bits, de modo que $M=$ $2^{4 k}$ representa a ordem da constelação. Usando o fato 
de que, constelações do tipo QAM (Quadrature-Amplitude Modulation) retangulares são geradas a partir de duas PAM (Pulse-Amplitude Modulation), é possível fazer uso deste modelo representativo em [17] para obter um novo conjunto de pontos em $\mathbb{H}$, geometricamente identificados no espaço quadrimensional $\mathbb{R}^{4}$, via isomorfismo natural definido em [16]

$$
s+x \mathbf{i}+y \mathbf{j}+z \mathbf{k} \stackrel{\mathbb{H} \simeq \mathbb{R}^{4}}{\longmapsto}(s, x, y, z) .
$$

De modo análogo às modulações já conhecidas, os pontos em cada um dos quatro eixos da modulação QuatMod distam de $2 d$ entre si. Assim, para uma constelação de $M$ pontos, cada eixo conterá os pontos

$$
\{d(-\sqrt[4]{M}+1), \ldots,-d, d, \ldots, d(\sqrt[4]{M}-1)\}
$$

$\mathrm{Na}$ Fig. 3 é apresentado um exemplo da modulação QuatMod para $M=16$. Como se pode perceber, todos os pontos ficam localizados em um hipercubo centrado na origem e de aresta igual a 2. Um hipercubo quadrimensional é uma extensão geométrica de um cubo de três dimensões, sendo fechado, compacto e conexo (i.e., dados quaisquer dois pontos, o segmento de reta determinado por esses pontos estará também na região do hipercubo) no sentido topológico.

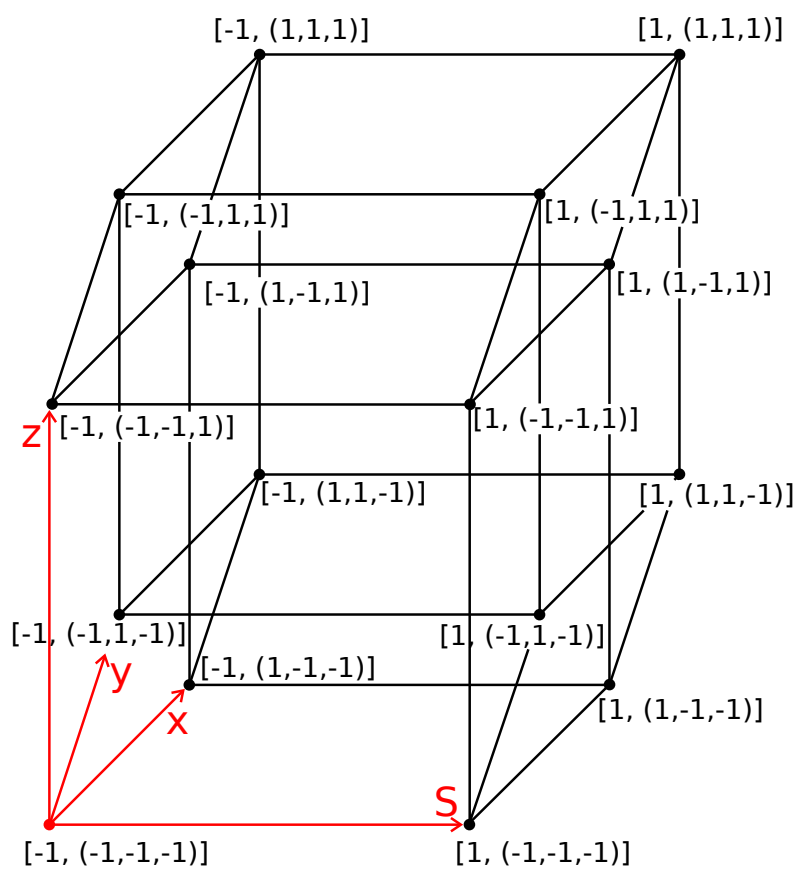

Fig. 3. Hipercubo $4 D$ projetado em $\mathbb{R}^{3}$

\section{B. Análise de Probabilidade de Erro em um Canal AWGN}

Neste novo modelo de modulação proposto, os símbolos estão dispostos em quatro dimensões ortogonais, assim, um símbolo $M$-QuatMod pode ser interpretado como quatro símbolos $\sqrt[4]{M}$-PAM independentes, que é uma modulação unidimensional, ou dois símbolos QAM, que é uma modulação bidimensional. Deste modo, a decisão correta, por máxima verossimilhança, do receptor por um símbolo QuatMod equivale decidir corretamente quatro símbolos PAM, ou seja,

$$
P_{M}^{Q u a t M o d}=1-\left(1-P_{\sqrt[4]{M}}^{P A M}\right)^{4}
$$

em que $P_{M}^{P A M}$ representa a probabilidade de erro de um símbolo $M$-PAM, definido em [17, eq. (5-2-45)] como

$$
P_{M}^{P A M}=\frac{2(M-1)}{M} \mathbf{Q}\left[\sqrt{\frac{6 \varepsilon_{a v}}{\left(M^{2}-1\right) N_{0}}}\right],
$$

em que $\varepsilon_{a v}$ representa a energia média do símbolo e $N_{0}$ denota a energia do ruído térmico.

Aplicando (15) em (16), obtém a probabilidade de erro de um símbolo $M$-QuatMod em um canal AWGN, que é dada por

$$
P_{M}^{Q u a t M o d}=1-\left(1-\frac{2(\sqrt[4]{M}-1)}{\sqrt[4]{M}} \mathbf{Q}\left[\sqrt{\frac{6 \gamma}{\sqrt{M}-1}}\right]\right)^{4},
$$

em que $\gamma=\varepsilon_{a v} / N_{0}$.

Comparando com o modelo descrito na seção III, um canal AWGN quaterniônico equivale a um ganho unitário nos canais copolares e a um ganho nulo aos canais de polarização cruzada, ou seja, $h_{h h}=h_{v v}=1$ e $h_{h v}=h_{v h}=0$.

\section{Resultados}

Nesta seção serão apresentados resultados de simulação, a fim de valiar os resultados teóricos apresentados no decorrer deste trabalho. Todas as simulações foram realizadas utilizando $10^{5}$ iterações de Monte Carlo. Além disso, considerou-se que os símbolos transmitidos eram gerados aleatoriamente através de uma distribuição uniforme e que no receptor o sinal recebido era contaminado com um ruído AWGN com média nula.

Na Fig. 4 é apresentado uma comparação entre os resultados simulados e teóricos de probabilidade de erro de um sinal transmitido utilizando as modulação 16 e 256-QuatMod. Nesta simulação um canal AWGN é considerado, bem como a potência dos símbolos quaterniônicos possui média unitária. Vale salientar que o ruído inserido é igualmente espalhado em todas as dimensões do sinal recebido. Como se pode perceber, a formulação teórica desenvolvida na eq. (17) está de acordo com os resultados simulados, comprovando assim sua validade.

$\mathrm{Na}$ Fig. 5 é realizada uma comparação entre as probabilidades de erro de símbolo do sistema de modulação QuatMod com o tradicional QAM em um canal AWGN. Nesta comparação, ambos os símbolos QuatMod e QAM possuem potência média unitária. Como se pode notar, os símbolos modulados em 16-QuatMod obtiveram um ganho de $4 \mathrm{~dB}$ em relação ao 16-QAM e obteve resultados bastante próximos da probabilidade de erro do 4-QAM. Já o 256-QuatMod, que possui 8 bits por símbolo, apresentou um desempenho próximo ao do 32-QAM, que possui 5. Deste modo, constata-se que a modulação QuatMod possui um capacidade de transmitir uma maior quantidade de informação para uma mesma taxa de erro se comparado com o QAM.

\section{CONCLUSÕES}

Neste artigo foi apresentada uma modelagem de canal equivalente quaterniônico utilizando a diversidade por 


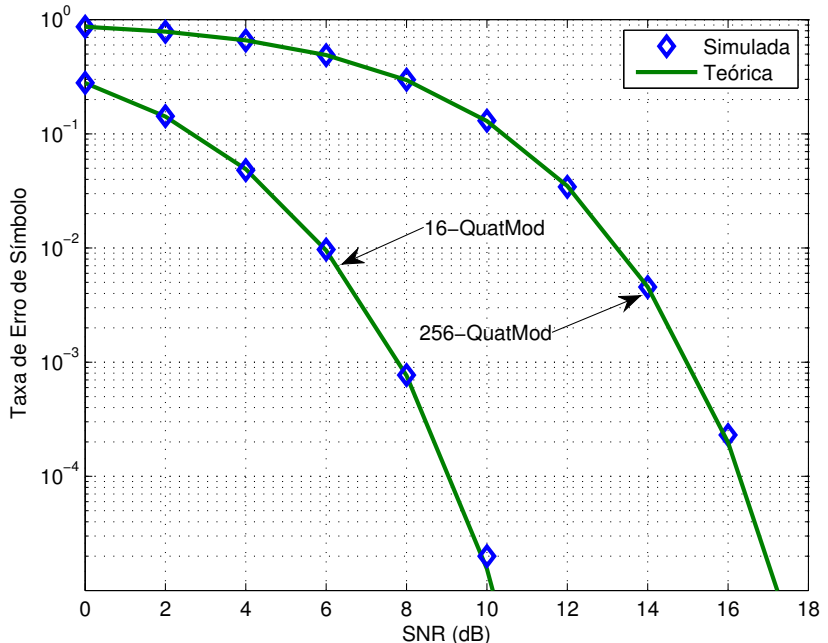

Fig. 4. Comparativo entre os resultados teóricos e simulados para o 16 e o 256 QuatMod

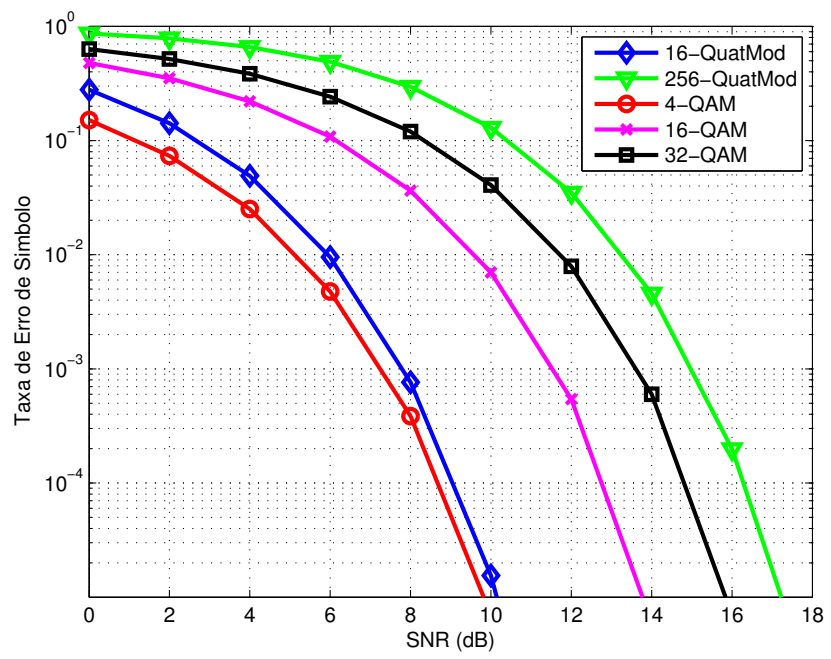

Fig. 5. Comparativo entre o QuatMod e o QAM

polarização. Foi também apresentada a técnica de modulação quaterniônica QuatMod bem como uma análise de sua probabilidade de erro de símbolo em um canal AWGN. $\mathrm{Na}$ seção $\mathrm{V}$ foram apresentados resultados de simulação comprovando a validade da formulação analítica proposta.

Como se pôde concluir neste trabalho, o modelo de canal quaterniônico apresenta-se como uma nova alternativa de modelagem de sistema, abrindo novas possibilidades para o processamento de sinais. Já o QuatMod apresentou resultados bastante satisfatórios de taxa de erro de símbolo, além de utilizar como recurso extra as propriedades intrínsecas das ondas eletromagnéticas na diversidade por polarização, não necessitando de instantes de tempo extras e tampouco de frequências adicionais, apresentando-se como uma técnica bastante promissora. Além disso, o processamento de um sinal QuatMod é feito de forma quaterniônica, o que aumenta a eficiência computacional.
Como perspectivas de trabalhos futuros podem se destacar: Analisar canais quaterniônicos com desvanecimento; Analisar sistemas com múltiplas antenas dual-polarizadas; Estudar sistemas de comunicação quaterniônicos com multi taxas de transmissão; Pesquisar sobre o uso de quatérnios em sistemas de comunicações com multiportadoras não ortogonais; Estudar conceitos de codificação de sinais em sistemas quaterniônicos; Generalizar o modelo quaterniônico para outros modelos hipercomplexos com dimensões superiores a quatro.

\section{REFERÊNCIAS}

[1] B. S. Collins, "Polarization-diversity antennas for compact base stations". Microwave Journal, Vol. 43, No 1, pp. 76-88, January 2000.

[2] R. U. Nabar, H. Bölcskei, V. Erceg, D. Gesbert, e A. J. Paulraj, "Performance of multiantenna signalling techniques in the presence of polarization diversity". IEEE Trans. Signal Processing, vol. 50, pp. 2553-2562, 2002.

[3] T. W. C. Brown, S. R. Saunders, S. Stavrou, e M. Fiacco, "Characterization of Polarization Diversity at the Mobile", IEEE Transactions on Vehicular Technology, vol. 56, no. 5, pag. 2440-2447, Setembro, 2007.

[4] T. A. Ell, "Hypercomplex spectral transforms". Ph.D. dissertation, Univ. Minnesota, Minneapolis, 1992.

[5] Soo-Chang Pei, Jian-Jiun Ding e Ja-Han Chang, "Efficient implementation of quaternion Fourier transform, convolution, and correlation by 2-D complex FFT". IEEE Transactions on Signal Processing, vol. 49, no. 11, pp. 2783-2797, Novembro, 2001.

[6] H. Yamamoto e N. Aoshima, "Three dimensional signal processing based on quaternion", Proceedings of the 41st SICE Annual Conference, vol. 3, pag. 1420-1424, Agosto, 2002

[7] B. J. Wysocki, T. Wysocki, e J. Seberry, "Modeling Dual Polarization Wireless Fading Channels using Quaternions", Computer \& Electronics Engineering Faculty Publications, n. 27, 2006.

[8] J. Seberry, K. Finlayson, S. S. Adams, T. A. Wysocki, Tianbing Xia e B. J. Wysocki, "The Theory of Quaternion Orthogonal Designs". IEEE Transactions on Signal Processing, vol. 56, no. 1, pp. 256-265, Janeiro. 2008.

[9] J. Gomes e L. Velho, "Computação Gráfica, volume". Série de Computação e Matemática. Rio de Janeiro, IMPA, 1998.

[10] N. Le Bihan e J. Mars, "Singular Value Decomposition of Quaternion Matrices: A new Tool for Vector-sensor Signal Processing". Signal Process., vol. 84, pp. 1177-1199, 2004.

[11] S. Miron, N. Le Bihan, e J.I. Mars, "Quaternion MUSIC for Vector-Sensor Array Processing". IEEE Transactions on Signal Processing, vol. 54, no. 4, pp. 1218-1229, Abril, 2006.

[12] C. Cheong-Took e D. P. Mandic, "A Quaternion Widely Linear Adaptive Filter”. IEEE Trans. Signal Process., vol. 58, pp. 4427-4431, 2010.

[13] P. Ginzberg e A. T. Walden, "Testing for quaternion propriety". IEEE Transactions on Signal Processing, vol. 59, pp. 3025-3034, 2011.

[14] P. Ginzberg e A. T. Walden, "Quaternion VAR Modelling and Estimation". Signal Processing, IEEE Transactions on , vol.61, no.1, pp.154-158, Janeiro, 2013.

[15] Kiupers, Jack B. "Quaternions and Rotations Sequences: A Primer with Aplications to Orbits, Aerospace and Vitual Reality". New Jersey, Princeton University Press, 1999.

[16] J. H. Conway e D. A. SMITH, "On Quaternions and Octonions: Their Geometry, Arithmetic, and Symmetry”. A K Peters/CRC Press, ed. 1, 2003.

[17] J. G. Proakis, "Digital Communications". McGraw-Hill Series in Electrical and Computer Engineering, ed. 3, 1995.

[18] O. M. Isaeva e V. A. Sarytchev, "Quaternion presentations polarization state", 2nd IEEE Topical Symposium of Combined Optical-Microwave Earth and Atmosphere Sensing, pp.195-196, Abril, 1995.

[19] I. P. Shkarofsky e S. B. Nickerson, "Multipath Depolarization Theory Combining Antenna with Atmospheric and Ground Reflection Effects". Annales des Télécommunications, vol. 36, no. 1-2, pp. 83-88, Jan-Fev, 1981.

[20] S. B. Nickerson e I. P. Shkarofsky, "A Computer Simulation of XPD Degradation Due to Multipath Propagation". Annales des Télécommunications, vol. 36, no. 1-2, pp. 89-94, Jan-Fev, 1981. 